\title{
Probing the Onset of Functional Behavior by Exciting Reversible Atom Displacements Using Variable Voltages and Dose Rates
}

\author{
C. Kisielowski ${ }^{1}$, L.-W. Wang ${ }^{2}$, P. Specht ${ }^{3}$, J.H. Kang ${ }^{4}$, R. Cieslinski ${ }^{4}$
}

1. Joint Center for Artificial Photosynthesis and National Center for Electron Microscopy, Lawrence Berkeley National Laboratory, One Cyclotron Rd., Berkeley CA 94720 USA

2. Material Sciences Division, Lawrence Berkeley National Laboratory, One Cyclotron Rd., Berkeley CA 94720 USA

3. Dept. of Mat. Sci. \& Eng., UC Berkeley, Berkeley CA 94720 USA

4. The DOW Chemical Company, Midland MI 48667 USA

In recent years the deployment of new electron-optical components such as aberration-correctors, monochromators, or high-brightness electron sources have greatly improved on capabilities to interrogate materials at the atomic level. Most noticeable is DOE's Transmission Electron Aberrationcorrected Microscope Project, which delivered deep sub-Ångstrom resolution and single atom sensitivity across the Periodic Table of Elements [1,2]. Beyond such extraordinary capablities, it was recently demonstrated that such equipment allows for atomic resolution imaging between $\mathrm{U}=20 \mathrm{kV}$ and $\mathrm{U}=$ $300 \mathrm{kV}$ [3]. Even the atomic structure of soft or hard matter can now be recovered at improved resolution using in-line holograms, which are reconstructed from images series that are recorded with ultra-low dose rates $<10^{-18}$ Ampere/ $/ \AA^{2}$ [3]. Exploring the capabilities that emerge from a rigorous current and voltage control, the Figure 1 points out that the incident power of the impingent electrons $P$ $=\mathrm{U}^{*} \mathrm{I}$ can be chosen such that irreversible (1a) or reversible (1b) object alterations can be stimulated in molecules, nanomaterials or in surface proximity that are not only driven by irreversible, voltage dependent ionization and displacement processes but also by reversible phonon excitations to a large extent [3,4]. Of particular interest is the intermediate zone (1c) between both extremes since it allows studying conformational object changes at atomic resolution as a result of an external stimulus. In fact, we find that dynamic and reversible contributions to contrast modifications in high-resolution images commonly exceed contributions from electron scattering at static objects where temperature is modeled by Debye-Waller factors, only [4]. In the past, contrast losses by $50 \%$ or more were known as unexplainable mismatches between experimental images and image simulations [7].

The low voltage performance of the TEAM0.5 microscope is still limited by lens aberrations as shown in Figure 2a). A peak performance value occurs because the image contrast increases with decreasing voltage as a result of the voltage dependent electron scattering process; the larger scattering angles at low voltages, however, require a more precise correction of residual lens aberrations to reach optimal performance, which is currently pursued in the Salve and the $\mathrm{C}^{3}$ Projects (Figure 2a). Dose rates (beam currents) greatly affect the material integrity through phonon excitations. However, they can be tuned to maintain the static object structure even if very few atoms are imaged (Figures $2 \mathrm{~b}$ ) or to reveal reversible object excitations $[3,4]$. We model beam-induced, collective atom displacements in ab-initio Molecular Dynamics simulations and find that many body effects are essential since they can temporarily stabilize energetically favorable object configuration that cannot be predicted by single atom trajectories [4]. Therefore, current and voltage control in electron microscopy enables imaging of single molecules and their conformations using a weak excitation approach that also reveals energetically preferred object configurations at atomic resolution, which describe the onset of functional behavior $[4,8]$. 
[1] N. Alem, N. et al. Phys. Rev. Lett. 2011, 106, 1261023

[2] C. Kisielowski et al. Microsc. Microanal. 2008, 14, 454-462

[3] B. Barton, Microsc. Microanal. 2012, 18, 982-994

[4] C. Kisielowski et al. J. American Chemical Society, 2013, submitted

[5] H. N. Chapman, P. Fromme, A. Barty, et al. Nature, 2011, 470, $72-78$

[6] R. Fung, V. Shneerson, D.K. Saldin, A. Ourmazd, Nature Physics, 2009, 5, 64-67

[7] A. Howie, Ultramicroscopy 2004, 98, 73-79

[8] Electron Microscopy was performed at NCEM, which is supported by the U.S. Department of Energy under Contract No. DE-AC02 - 05CH11231. The Dow Chemical Company supported P. Specht for the investigations of rhodium-based catalysts.

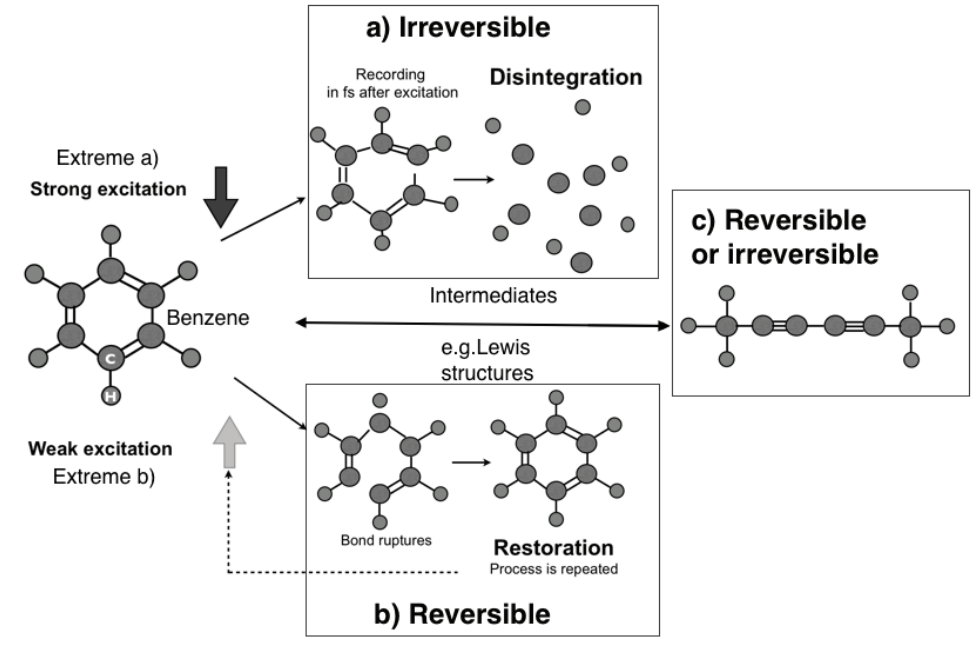

a) Voltage variation - Contrast gain
Figure 1. Schematic representation of reversible and irreversible system excitations:

a) Strong excitation [5], b) Weak excitation $[4,6]$ c) An intermediate experiment [4]. For simplicity a benzene ring is depicted but the schematics applies to any atom cluster on an undefined substrate.

Figure 2. a) Contrast gain by voltage variation between 20 and $300 \mathrm{kV}$. Experiments: silicon atoms in a wedge shaped sample [4], Simulation: one tungsten atom. b) Gaining object stability by current reduction. A cluster of 7 tungsten atoms on alumina is shown.

b) Current variation - Stability gain

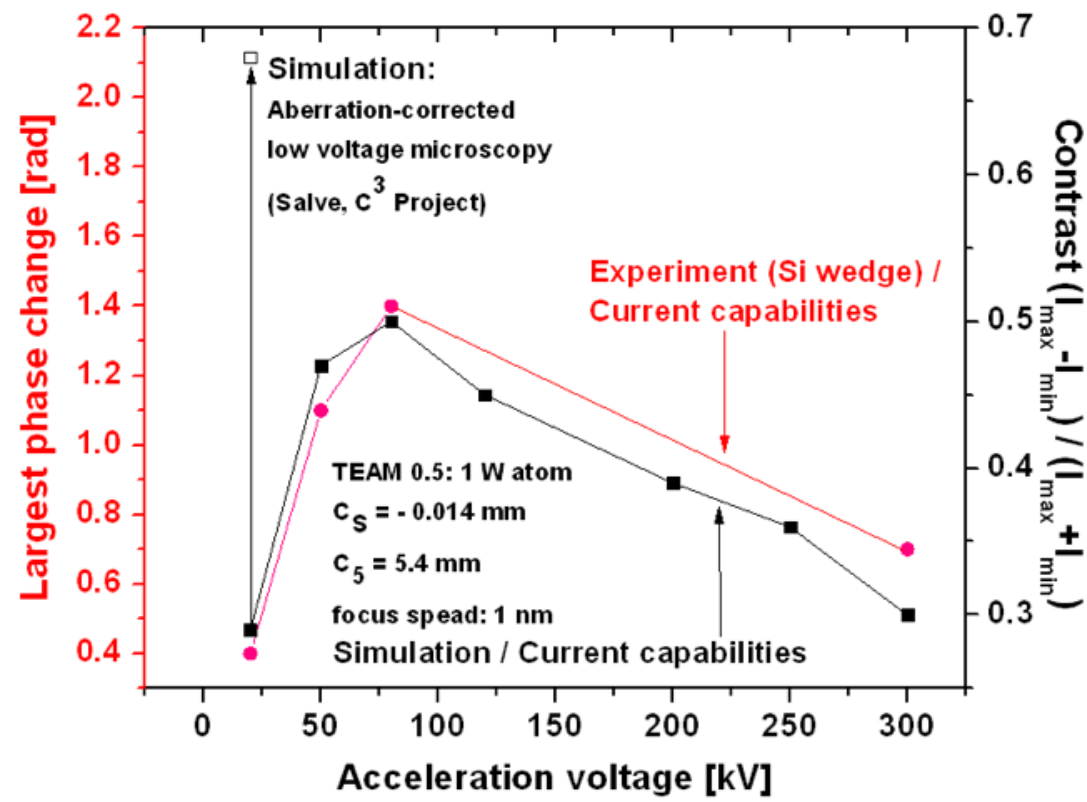

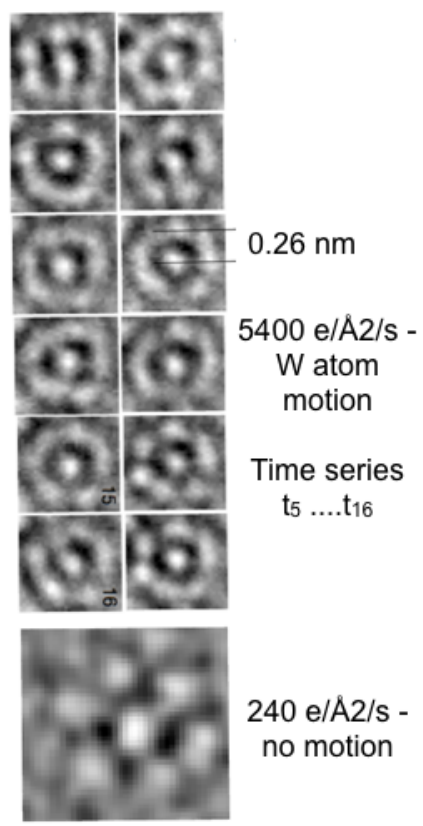

